\title{
A DWARF NOVA IN THE GLOBULAR CLUSTER M13
}

\section{Citation}

Servillat, M., N. A. Webb, F. Lewis, C. Knigge, M. van den Berg, A. Dieball, and J. Grindlay. 2011. "A DWARF NOVA IN THE GLOBULAR CLUSTER M13." The Astrophysical Journal 733 (2): 106. https://doi.org/10.1088/0004-637x/733/2/106.

\section{Permanent link}

http://nrs.harvard.edu/urn-3:HUL.InstRepos:41399766

\section{Terms of Use}

This article was downloaded from Harvard University's DASH repository, and is made available under the terms and conditions applicable to Other Posted Material, as set forth at http:// nrs.harvard.edu/urn-3:HUL.InstRepos:dash.current.terms-of-use\#LAA

\section{Share Your Story}

The Harvard community has made this article openly available.

Please share how this access benefits you. Submit a story.

\section{Accessibility}




\title{
A DWARF NOVA IN THE GLOBULAR CLUSTER M13
}

\author{
M. Servillat ${ }^{1}$, N. A. WebB ${ }^{2,3}$, F. Lewis ${ }^{4,5}$, C. KNigGE ${ }^{6}$, M. van Den Berg ${ }^{1,7}$, A. Dieball ${ }^{6}$, And J. GRindlay ${ }^{1}$ \\ ${ }^{1}$ Harvard-Smithsonian Center for Astrophysics, 60 Garden Street, MS-67, Cambridge, MA 02138, USA; mservillat@ cfa.harvard.edu \\ ${ }^{2}$ Université de Toulouse; Université Paul Sabatier-Observatoire Midi-Pyrénées, Institut de Recherche en Astrophysique et Planétologie (IRAP), Toulouse, France \\ ${ }^{3}$ Centre National de la Recherche Scientifique; IRAP; 9 Avenue du Colonel Roche, BP 44346, F-31028 Toulouse cedex 4, France \\ ${ }^{4}$ Department of Physics and Astronomy, The Open University, Walton Hall, Milton Keynes, MK7 6AA, UK \\ ${ }^{5}$ Faulkes Telescope Project, University of Glamorgan, Pontypridd, CF37 4BD, UK \\ ${ }^{6}$ Department of Physics, University of Southampton, Southampton, S017 1BJ, UK \\ ${ }^{7}$ Department of Physics and Astronomy, Utrecht University, 3508 TC Utrecht, The Netherlands \\ Received 2011 January 13; accepted 2011 March 21; published 2011 May 12
}

\begin{abstract}
Dwarf novae (DNe) in globular clusters (GCs) seem to be rare with only 13 detections in the 157 known Galactic GCs. We report the identification of a new DN in M13, the 14th DN identified in a GC to date. Using the $2 \mathrm{~m}$ Faulkes Telescope North, we conducted a search for stars in M13 that show variability over a year (2005-2006) on timescales of days and months. This led to the detection of one DN showing several outbursts. A Chandra X-ray source is coincident with this DN and shows both a spectrum and variability consistent with that expected from a DN, thus supporting the identification. We searched for a counterpart in Hubble Space Telescope Advanced Camera for Surveys/Wide Field Camera archived images and found at least 11 candidates, of which we could characterize only the 7 brightest, including one with a $3 \sigma \mathrm{H} \alpha$ excess and a faint blue star. The detection of one DN when more could have been expected likely indicates that our knowledge of the global Galactic population of cataclysmic variables is too limited. The proportion of DNe may be lower than found in catalogs, or they may have a much smaller mean duty cycle $(\sim 1 \%)$ as proposed by some population synthesis models and recent observations in the field.
\end{abstract}

Key words: globular clusters: individual (M13, NGC 6205) - novae, cataclysmic variables - stars: dwarf novae $\mathrm{X}$-rays: general

Online-only material: color figures

\section{INTRODUCTION}

Globular clusters (GCs) are old, gravitationally bound stellar systems with extremely high stellar densities in their core which enable cluster members to dynamically interact through regular encounters. Close binary stars have been suggested to play a significant role in the dynamical evolution of GCs (Hut et al. 1992). Encounters between passing stars and close binaries may harden the latter and accelerate the passing star. This leads to the formation of a variety of tight and interacting binaries, and by accelerating stars in the core, close binaries may delay or halt the inevitable gravitational core collapse of GCs.

Many of the X-ray sources in GCs discovered in the 1970s have been resolved into a multitude of close binaries and related systems with XMM-Newton and Chandra (see, e.g., Servillat et al. 2008a, 2008b). These include active and quiescent lowmass X-ray binaries (LMXBs) with a neutron star (NS) primary, millisecond pulsars (MSPs), cataclysmic variables (CVs), and magnetically active binaries. The number of NS LMXBs in GCs as well as part of the CV population appears to scale with the stellar encounter rate of their host cluster, indicating that they are indeed produced through dynamical mechanisms (Gendre et al. 2003; Pooley et al. 2003; Pooley \& Hut 2006). The same correlation is observed with MSPs in GCs detected in radio (Hui et al. 2010), and even in $\gamma$-rays (Abdo et al. 2010).

CVs are semi-detached binary stars with orbital periods typically of the order of hours, consisting of a white dwarf primary accreting via Roche lobe overflow from a companion which is usually a late-type, generally main-sequence star. Subtypes of CVs include non-magnetic CVs where an accretion disk forms around the primary, and magnetic systems where the white dwarf magnetic field is strong enough to disrupt (polar CVs, $B>10^{7} \mathrm{G}$ ) or truncate (intermediate polars, $10^{5}<B<10^{7} \mathrm{G}$ ) the accretion disk, and the mass flow is then channeled along the magnetic field lines (e.g., Cropper 1990; Patterson 1994). For systems with an accretion disk, it is believed that a thermal instability (e.g., Osaki 1996) is the cause of repetitive outbursts observed in CVs called dwarf novae (DNe). Optically, DN eruptions have amplitudes of 2-6 mag in $\mathrm{V}$, a duration of a few to 20 days and a recurrence timescale of weeks to years (e.g., Osaki 1996).

From the Catalog and Atlas of Cataclysmic Variables (Downes et al. 2001, updated 2006), DNe are the most common CVs identified in the field, with about half of the identified CVs being DNe. However, the sample of known field CVs suffers from various selection effects (Pretorius et al. 2007) and is biased toward bright and highly variable systems, such as DNe. We note that the distribution of those CVs is almost isotropic, which would indicate that the known population is mainly local (less than $1 \mathrm{kpc}$ around the Sun). The global Galactic population and its intrinsic composition is thus not fully understood.

Population synthesis models indicate that a fainter population showing fewer outbursts may dominate the population of $\mathrm{DNe}$ (up to 70\%; Kolb 1993; Howell et al. 1997; Willems et al. 2005). Recent detections in the SDSS of faint CVs with short periods showed one of the first hints for the existence of such a population (Gänsicke et al. 2009), but its proportion is still not constrained.

If we limit our knowledge to the locally known population of CVs, there appear to be fewer DN outbursts from CVs in GCs (Shara et al. 1996; Dobrotka et al. 2006). This would mean that the population of CVs in GCs is intrinsically different from the known population in the field. However, it is clear that the known population in the field is not a reliable reference. We 
Table 1

Faulkes Telescope North $U$-band Observations

\begin{tabular}{llcr}
\hline \hline Epoch & \multicolumn{1}{c}{ Date } & MJD & Exposures \\
\hline 1 & 2005 Feb 18 & 53419 & $60 \mathrm{~s}, 120 \mathrm{~s}$ \\
& 2005 Feb 22 & 53423 & $120 \mathrm{~s}$ \\
2 & 2005 Mar 2 & 53431 & $3 \times 120 \mathrm{~s}$ \\
& 2005 Mar 4 & 53433 & $120 \mathrm{~s}$ \\
3 & 2005 Apr 12 & 53472 & $8 \times 120 \mathrm{~s}$ \\
& 2005 Apr 15 & 53475 & $9 \times 120 \mathrm{~s}$ \\
& 2005 Apr 16 & 53476 & $9 \times 120 \mathrm{~s}$ \\
& 2005 Apr 17 & 53477 & $9 \times 120 \mathrm{~s}$ \\
4 & 2005 May 5 & 53495 & $2 \times 800 \mathrm{~s}$ \\
5 & 2006 Mar 12 & 53806 & $44 \times 200 \mathrm{~s}$ \\
\hline
\end{tabular}

note that Shara et al. (1996) based their comparison on a small sample of about 20 well studied DNe which are not likely to represent the global population. Two hypotheses remain to be fully tested. (1) Dynamical effects and a specific environment in GCs (low-metallicity, long timescales) could shape differently the population of CVs. For example, it has been proposed that CVs in GCs are dominated by moderately magnetic systems (intermediate polars) with low accretion rates which show fewer outbursts (Dobrotka et al. 2006; Grindlay 1999). Also, typical masses of the accreting primaries may be higher, as massive white dwarfs can be more efficiently placed in binaries by tidal capture and three-body processes (Ivanova et al. 2006). (2) The lack of DN outbursts from CVs in GCs could be due to detection biases. Due to the crowding in optical, DN outbursts are indeed more difficult to catch in GCs (Pietrukowicz et al. 2008). These questions can only be solved with further observations and comparisons with more complete populations of $\mathrm{CVs}$, both in the field and in GCs.

We present here a search for optically variable stars, and particularly DN outbursts, in the GC M13 (NGC 6205). From the catalog of Harris (1996, updated in 2010), M13 is at a distance of $7.1 \mathrm{kpc}$ from the Sun. Its core radius is $0{ }^{\prime} 62$, which corresponds to the half-width at half-maximum of the luminosity profile of the cluster, and its half-mass radius is 1'.69 (region encircling half of the mass, approximated to be half of the light from the cluster). M13 is a low-metallicity cluster with $[\mathrm{Fe} / \mathrm{H}]=-1.53$. The reddening along the line of sight is $E(B-V)=0.02$, which corresponds to a low extinction $A_{V} \sim 0.06$ and an absorption from a column density of $N_{\mathrm{H}} \sim 1.1 \times 10^{20} \mathrm{~cm}^{2}$.

We first present our search for optical variables and outbursts (Section 2). We then detail Chandra X-ray observations of M13 used to search for X-ray sources coincident with the optically variable ones (Section 3). In Section 4, we describe one peculiar optical event aligned with an X-ray source and present archived Hubble Space Telescope (HST) data to uncover possible counterparts. Finally, we discuss the results in Section 5.

\section{OPTICAL OBSERVATIONS}

\subsection{Data and Reduction}

The GC M13 has been observed with the Faulkes Telescope North (FTN) at Haleakala on Maui, USA, during the nights indicated in Table 1. The FTN is a $2 \mathrm{~m}$ robotic telescope optimized for research and education (Roberts et al. 2008; Lewis \& Roche 2009) and is part of the Las Cumbres Observatory Global Telescope Network.

Imaging was obtained in $U, B, V, R, i, \mathrm{H} \alpha$, and OIII filters between 2005 February and 2006 April. We focus here on the $U$ band as this data set is the cleanest and the most complete, and CVs are generally blue objects so they are likely to be among the brightest objects compared to GC main-sequence stars in the $U$ band. Moreover, narrowband filters are not as deep, and we obtained fewer images in the other filters.

The DillCam CCD detector we used has $2048 \times 2048$ pixels binned $2 \times 2$ prior to readout into effectively $1024 \times 1024$ pixels. The field of view is $4.7 \times 4^{\prime} .7$ and the pixel scale is $0^{\prime \prime} .278$ pixel $^{-1}$. Automatic pipelines de-bias and flat-field the Faulkes science images using calibration files from the beginning and the end of each night.

The seeing ranged from $1^{\prime \prime} .2$ to more than $3^{\prime \prime}$. We discarded images with poor signal-to-noise ratio $(\mathrm{S} / \mathrm{N}$; mostly due to thin clouds) and images with tracking problems (where the stars are non-spherical and stretch more than a few pixels during the exposure), an excess of hot pixels, or a background gradient across the image. Finally, $78 U$-band images were kept. They can be grouped into five epochs of observations, each epoch lasting one to a few days. The first four epochs were taken over two and a half months and the fifth epoch about one year later.

\subsection{Search for Variability}

We looked for variable stars in M13 using difference imaging photometry. First, we aligned the images by selecting three stars in a reference image. The task imregister under IRAF ${ }^{8}$ was used to get a set of $1024 \times 1024$ pixel images all aligned on the reference image after rotation and translation. Due to this method, the edges of some images were cut, and the usable field of view for this search has been reduced to $\sim 4^{\prime} \times 3$. 7 , which still included the half-mass radius of M13.

We computed a reference image by taking the median of five images with the best seeing (about $1^{\prime \prime} .2$ ). This image was then subtracted from all the other images using the ISIS 2 software (Alard 2000). This tool uses adaptive kernel techniques (Alard \& Lupton 1998) and gives optimal results in the case of crowded fields, variable seeing, and point-spread function (PSF). We set the kernel size to $9 \times 9$ and the stamp size to $15 \times 15$ pixels (the stamp size is the area taken by the program around each object for computation). Three Gaussians were used in the convolution. The background is nearly flat so it was fitted with a constant. The spatial variations in the PSF kernel were modeled by a 4th order polynomial. After this process, the mean of the obtained images was close to zero, as expected, with some isolated positive or negative sources.

For each subtracted image, we detected variable star candidates using IDL procedures from the Astrolib ${ }^{9}$ (e.g., find). We estimated the background level and the standard deviation $\sigma$ in an annulus around the source and performed a detection for sources with a significance higher than $5 \sigma$. However, when irregular features were present in the subtracted images, the IDL/Astrolib find procedure gave a high number of false detections. When this was the case, all the detections for that image were ignored. We thus selected the most secure detections and possibly missed low significance events. We finally merged the different lists and found a total of 16 variable star candidates in the field of view. They are listed in Table 2.

For each detected variable star in each subtracted image, we first fitted the star with a Gaussian and estimated the integrated

\footnotetext{
8 IRAF is distributed by the National Optical Astronomy Observatory, which is operated by the Association of Universities for Research in Astronomy, Inc., under cooperative agreement with the National Science Foundation

9 http://idlastro.gsfc.nasa.gov/
} 


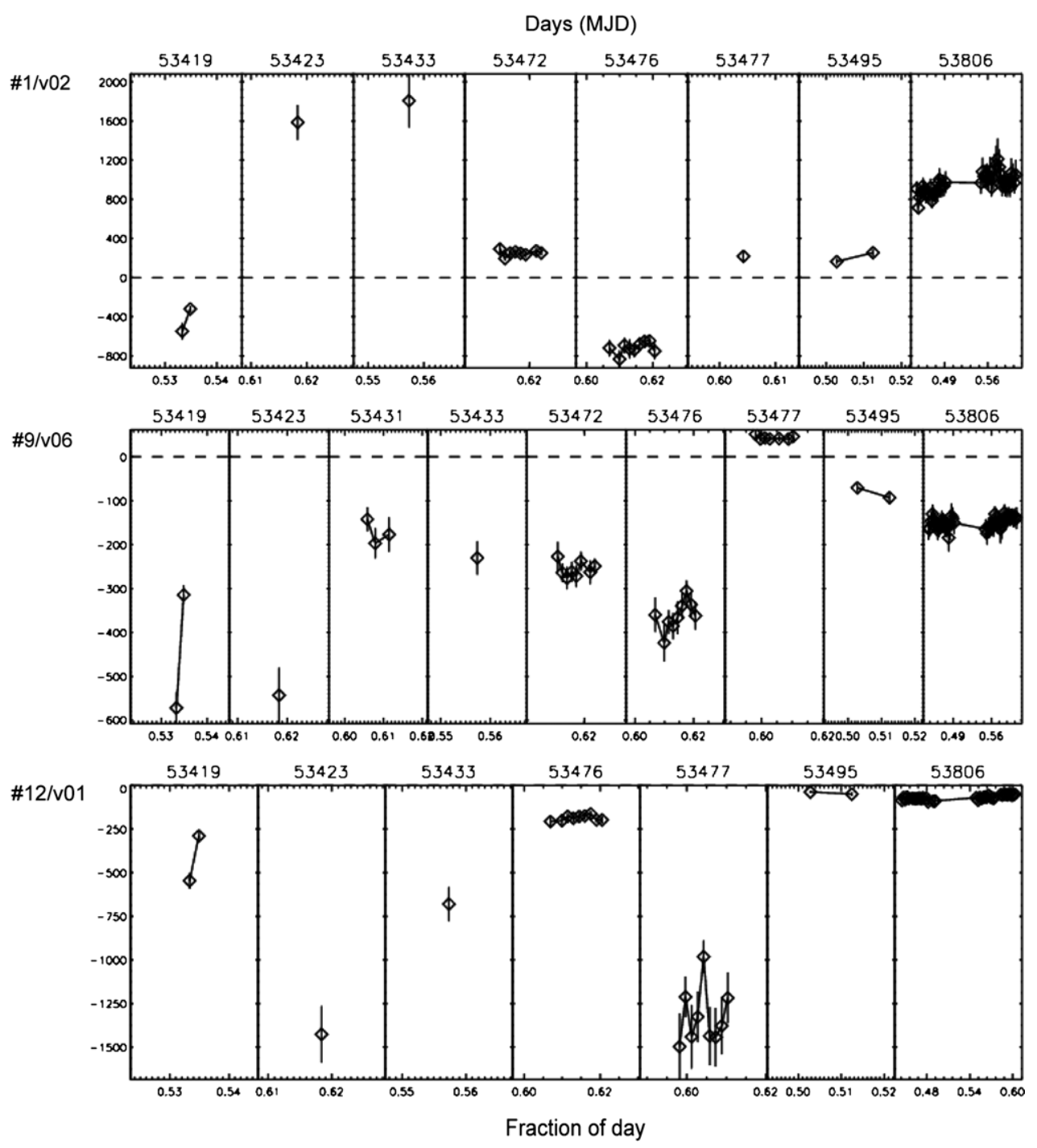

Figure 1. Light curves of three variable star candidates associated with BL Her variables; see Table 2. The $y$-axis gives the flux relative to the reference image in arbitrary units with $1 \sigma$ error bars.

flux in arbitrary units. The typical observed full width at halfmaximum (FWHM) value in the subtracted images is about $1^{\prime \prime} .5$, slightly larger than the FWHM of the reference image. We estimated the significance of the variable star candidates by comparing their amplitude with the standard deviation of the surrounding background. For the light curve extraction, we kept only measurements that were higher than three times the standard deviation for each variable star. Some examples of light curves are shown in Figures 1-4. The zero level in those plots corresponds to the flux of the star in the reference image. If no point is reported for a given day, it is either because the luminosity of the object is at the same level as in the reference image (then no emission is seen in the subtracted image) or the emission is not significant $(<3 \sigma)$ compared to the surrounding background of the subtracted image. We thus have discarded some low significance measurements.

In order to check if our method could lead to false positives, we added manually five entries in the variable star candidate list (aligned with bright stars or randomly placed in the image). The chosen positions did not correspond to objects detected as variables. We applied the same extraction process for all five positions and we did not obtain any significant measurements, i.e., we did not obtain false detections.

\subsection{Known Variable Stars}

In order to compare our list of variable stars with previous catalogs, we added an astrometric solution to the reference image by aligning stars using the 2MASS Catalog (Skrutskie et al. 2006) which provided 15 good reference stars. We used GAIA $^{10}$ to fit the position of those stars and generate an astrometric solution for the reference image with an rms error of $0^{\prime \prime} 2$. Taking into account the accuracy of 2MASS, the total astrometry over the field of view is 0.3 ( $1 \sigma$ error).

We compared our variable star list to the Catalog of Variable Stars in Globular Clusters (Clement et al. 2001; Kopacki et al. 2003). We note that, as the subtracted images are convolved (and thus smoothed) during the subtraction process, the positional accuracy can be lower than for the reference image. We iteratively cross-matched the two lists to find a systematic offset. This bore-sight correction amounts to 0.93 and 0".66 in R.A. and decl., respectively, with a $1 \sigma$ error of 0.15 between the

${ }^{10}$ GAIA was created by the now closed Starlink UK project and has been more recently supported by the Joint Astronomy Centre Hawaii funded again by the Particle Physics and Astronomy Research Council (PPARC) and more recently by its successor organization the Science and Technology Facilities Council (STFC). 


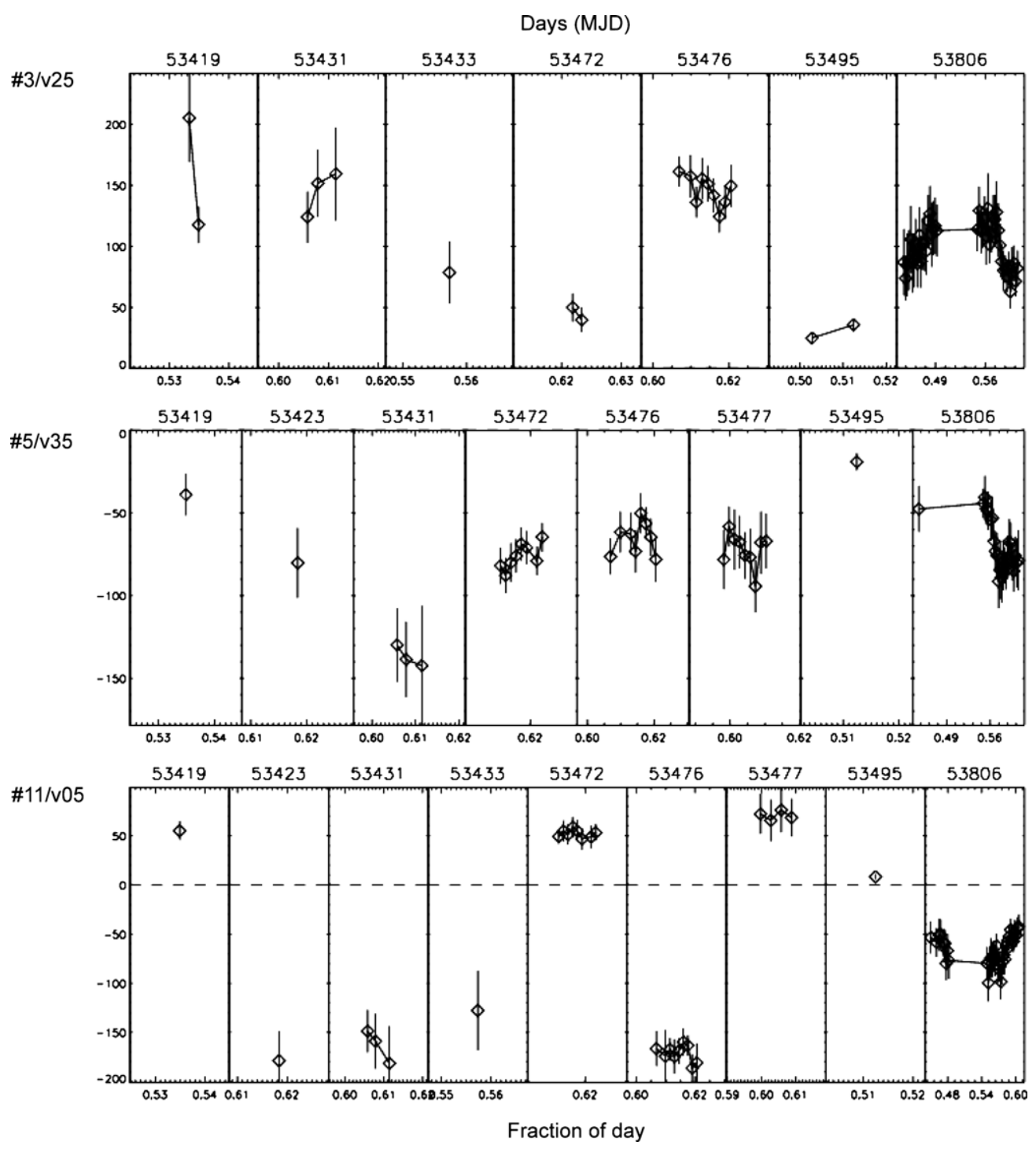

Figure 2. Light curves of three variable star candidates associated with RR Lyr stars; same comments as in Figure 1.

two sets, which adds up to the astrometric error. This led to the identification of 14 variable star candidates as already known variables. The name, type, period, and magnitude of the known variable stars are reported in Table 2. The 16 variable stars detected in this study as well as the known variables stars and the X-ray sources in the field of view are shown in Figure 5.

Although our data are insufficient to determine a period, the variability observed for stars 1 (v02) and 9 (v06) presented in Figure 1 is consistent with the previously observed periods (Kopacki et al. 2003). The same trend is seen for star 3 (v25) in Figure 1 with a shorter period of 0.3 day and all the other light curves extracted.

Red giant (RG) stars have previously been observed with semi-regular or irregular variability (Kopacki et al. 2003). The light curves we obtained for this category of candidates are consistent with this observation (Figure 3 ) but due to our limited sampling they could as well be periodic.

The variable star 7 is a known $U$ and $V$ bright object (misleadingly called UV-bright by Zinn et al. 1972), associated with M13 based on radial velocity measurements. The $U$ and $V$ bright stars referred to by Zinn et al. (1972) are in fact related to post-horizontal branch stars when plotted in a color-magnitude diagram (CMD; see their Figure 2). Star 7 corresponds to L993 and IV-52 in the catalogs of Ludendorff (1905) and Arp (1955), respectively, and has a spectral type A5III-IV from SIMBAD. ${ }^{11}$ This star has been reported as possibly variable (Meinunger 1978 ) but needed confirmation. No variability was reported by Kopacki et al. (2003, star labeled c), but we show its variability in this study, which seems to occur on timescales of a few days.

Finally, stars 2 and 4 have no previous detections as variable stars in the optical. Those stars are unlikely to be periodic variable stars with periods 0.2 to 8 days as previous, more complete searches for such periodic stars did not detect them (e.g., Kopacki et al. 2003). However, we note that the star 2 is variable over short timescales of 1 or 2 days, which seems quite short compared to typical DN outbursts. In order to constrain the nature of those new events, we searched for possible X-ray counterparts, as reported in the next section.

\section{Chandra X-RAY OBSERVATION OF M13}

In the Chandra X-ray Observatory archive, we found two observations of M13 with the ACIS-S detector on 2006 March 10

\footnotetext{
11 http://simbad.u-strasbg.fr/
} 


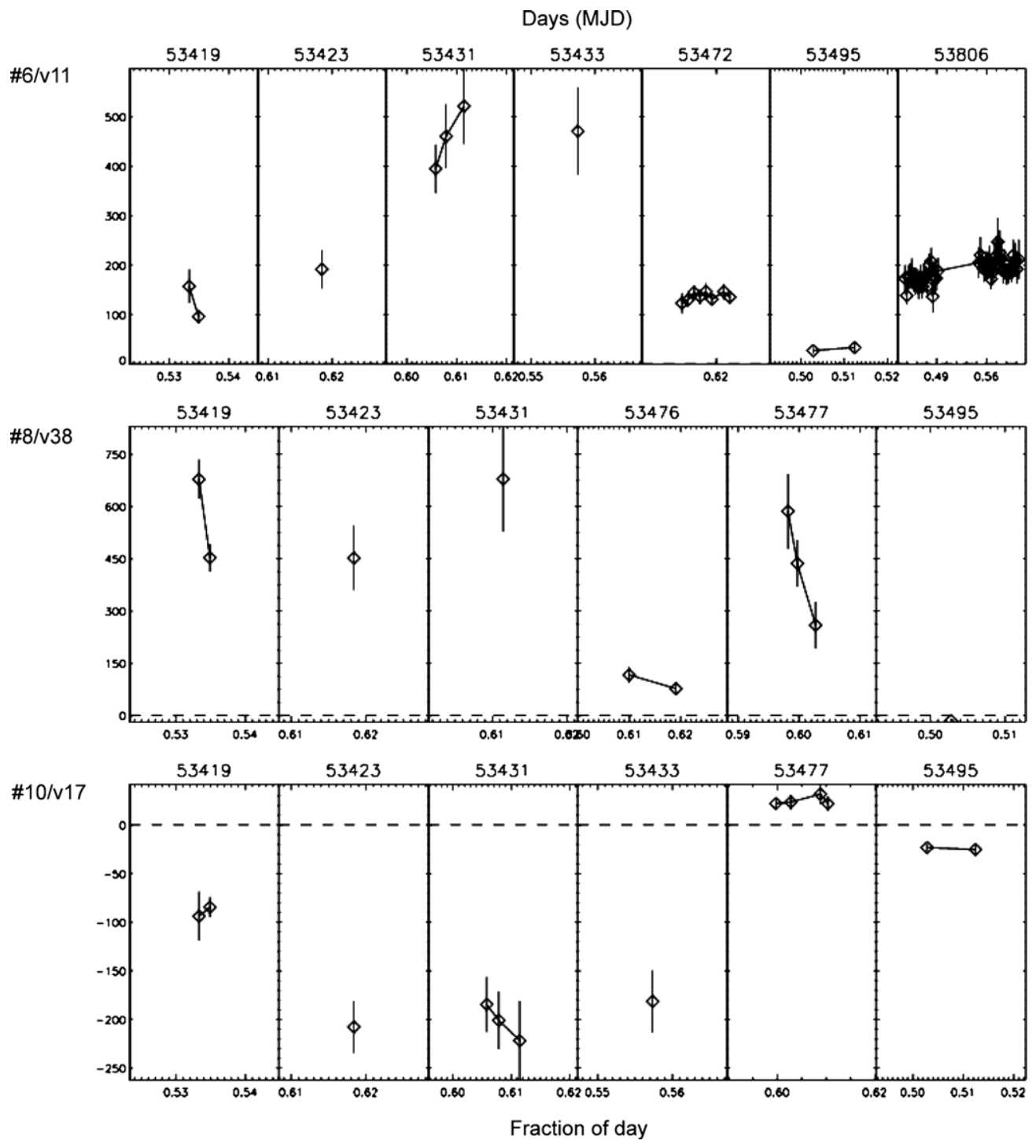

Figure 3. Light curves of three variable star candidates associated with RG stars; same comments as in Figure 1.

and 11 (MJD 53804-53805) for 27 and $28 \mathrm{ks}$ with a gap of $\sim 80$ ks between the observations (ObsID 7290 and 5436).

We reprocessed these data sets with CIAO 4.1 and combined the two data sets with the script merge_all. We then used wavdetect to detect sources in the energy ranges $0.5-2$ and a broader $0.3-8 \mathrm{keV}$ band with scales of 1.0, 1.4, 2.0, 2.8, 4.0, and 5.6 pixels. We selected a threshold probability of $10^{-6}$, designed to give one false source per $10^{6}$ pixels. We found 47 $\mathrm{X}$-ray sources in a $9^{\prime} \times 9^{\prime}$ region centered on M13 (Table 4). Two sources are only detected in the energy range $0.5-2 \mathrm{keV}$ (X14 and X30) and 12 are only detected in the broad band (including $\mathrm{X} 3, \mathrm{X} 10$, and $\mathrm{X} 13$ inside the half-mass radius). This list includes the 14 sources detected previously with XMM-Newton (Gendre et al. 2003) and available in the 2XMM catalog (Watson et al. 2009). An image that covers the half-mass radius of M13 is shown in Figure 6. A hardness ratio diagram is given in Figure 7.

In the energy range $0.5-2 \mathrm{keV}$, a total of 15 sources are located inside the half-mass radius and 8 of them are inside the core radius. Within a circle with a radius of 4.2 , we detected 35 sources (0.5-2 keV band). Using the study of Hasinger et al. (2005), which gives an estimate of the space density of background sources (active galactic nuclei, AGNs, in the soft $\mathrm{X}$-ray band $0.5-2 \mathrm{keV}$ ), we estimated the expected number of
AGNs for several regions (see Table 3). We assumed that the minimum flux of a detected source during the observation corresponds to 5 counts. A clear excess of detections is observed in the direction of M13. We limited this study to a region of radius $4{ }^{\prime} .2$ where the sensitivity drops to $86 \%$ of the central sensitivity and the PSF is slightly more elongated. As we assumed a constant minimum flux in this region, this could have led to a possible deficit of detections compared to the expected number of background sources in the larger region. However, this deficit is not observed, strengthening the excess of detections observed which may thus be slightly underestimated. As M13 is at a Galactic Latitude of 40.91 we expect only few foreground X-ray sources. Therefore, most of the X-ray sources inside the half-mass radius are likely to be associated with M13, and a tentative estimate of $19.6 \pm 3.9$ sources could be associated with the cluster.

\section{DETAILED RESULTS FOR THE VARIABLE STAR 4}

\subsection{X-ray Counterpart: Light Curve and Spectrum}

The star 4 falls in the error radius of an X-ray source detected with XMM-Newton on 2002 January 28 and 30 (Gendre et al. 2003). The X-ray source is described as variable by at least a factor $\sim 2$ on timescale of years by Gendre et al. (2003) as 


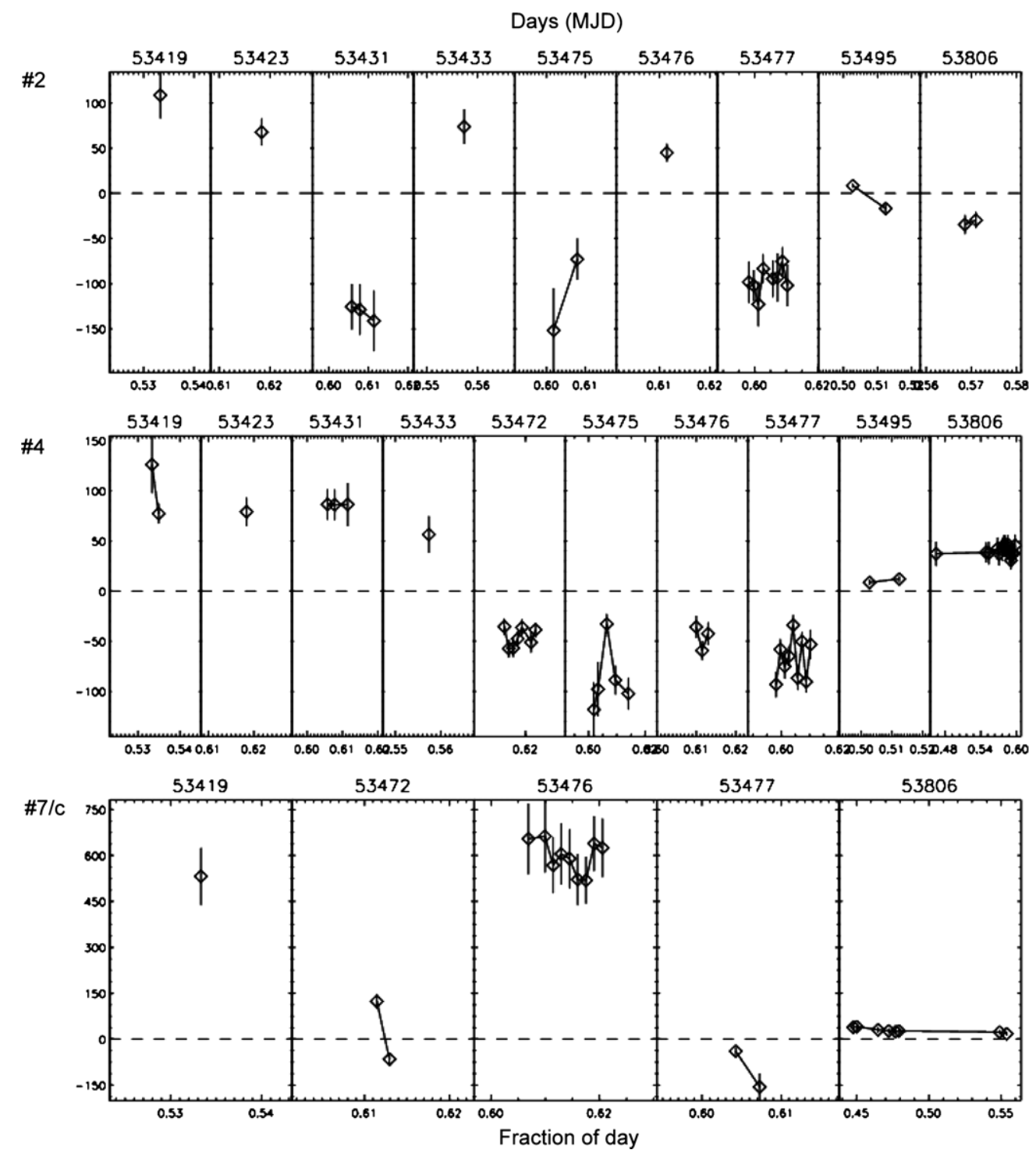

Figure 4. Light curves of three variable star candidates not associated with previously known variable stars; see Figure 1. Previously, star 7 had only been suspected to be variable.

it should have been detected by previous X-ray observations with ROSAT in 1994 (Verbunt 2001). The 2XMM catalog (Watson et al. 2009) gives a flux in the band $0.2-12.0 \mathrm{keV}$ of $3.9 \pm 0.7 \times 10^{-14} \mathrm{erg} \mathrm{s}^{-1} \mathrm{~cm}^{-2}\left(8.8 \times 10^{31} \mathrm{erg} \mathrm{s}^{-1}\right.$ at the distance of M13).

The position of the corresponding Chandra source (X6, see Section 3) is R.A. $=16^{\mathrm{h}} 41^{\mathrm{m}} 42^{\mathrm{s}} .48$, decl. $=+36^{\circ} 28^{\prime} 07^{\prime \prime} .6$ (J2000). We computed the $95 \%$ error from the formula given by Hong et al. (2005) and found 0.'32. Including the absolute pointing error of Chandra ACIS-S (0.7 at 95\%) we find a total error on the position of 0.77 at $95 \%$ confidence level. The variable star 4 lies $1^{\prime \prime}$. 15 away with a positional error of $1^{\prime \prime} .2$ at $95 \%$.

We extracted the light curve of source X6 from the Chandra data using the CIAO tool dmextract (see Figure 8). The count rate of source X6 was higher by a factor $\sim 3$ at the beginning of the observation and for $\sim 10 \mathrm{ks}$. The background is negligible throughout the observation.

We extracted the spectra of X6 for both observations using the tool psextract. We generated the RMF response files separately with the tool mkacisrmf. We used Sherpa to fit both spectra simultaneously, excluding the first $10 \mathrm{ks}$ of data. Given the limited number of counts (a total of 134 counts), we tried simple models with a fixed absorption of $1.1 \times 10^{20} \mathrm{~cm}^{-2}$ to fit the spectrum. We used the TBabs model with abundances from Wilms et al. (2000). Good fits were obtained for a power law with an index of $1.3 \pm 0.3\left(\chi^{2} /\right.$ dof $\left.=3.9 / 7\right)$ or a bremsstrahlung model with a temperature of $\mathrm{k} T \sim 35 \mathrm{keV}\left(\chi^{2} / \mathrm{dof}=4.2 / 7\right)$. The temperature is however not well constrained $(\mathrm{k} T<50 \mathrm{keV}$ at $1 \sigma)$. The spectrum and best fit are reported in Figure 9.

Based on the power-law models, we estimated that the flux of the source in the band $0.3-8.0 \mathrm{keV}$ (with $1 \sigma$ error) for the second observation is $(2.2 \pm 0.8) \times 10^{-14} \mathrm{erg} \mathrm{s}^{-1} \mathrm{~cm}^{-2}$ $\left(5.0 \times 10^{31} \mathrm{erg} \mathrm{s}^{-1}\right.$ at the distance of M13). During the increase of counts in the first observation, the flux reached $(5.2 \pm 1.9) \times 10^{-14} \mathrm{erg} \mathrm{s}^{-1} \mathrm{~cm}^{-2}\left(1.1 \times 10^{32} \mathrm{erg} \mathrm{s}^{-1}\right.$ at the distance of M13).

Due to the low number of counts, we estimated the median energy at three different time intervals to look for possible spectral variability (see Hong et al. 2004 for error estimations). During the first $\sim 10 \mathrm{ks}$, the median energy is $1.29 \pm 0.12 \mathrm{keV}$, then it rises to $1.77 \pm 0.25 \mathrm{keV}$ at the end of the first observation, 
Table 2

List of Variable Stars

\begin{tabular}{lccccccc}
\hline \hline No. & $\begin{array}{c}\text { R.A. } \\
\text { (h:m:s) }\end{array}$ & $\begin{array}{c}\text { Decl. } \\
\text { (d:m:s) }\end{array}$ & Name & Type & $\begin{array}{c}\text { Period } \\
\text { (day) }\end{array}$ & $\langle V\rangle$ & $\Delta V$ \\
\hline 1 & $16: 41: 35.90$ & $36: 27: 48.7$ & v02 & BL Her & 5.11 & 13.01 & 0.87 \\
2 & $16: 41: 44.57$ & $36: 28: 04.4$ & & & & & \\
3 & $16: 41: 42.73$ & $36: 27: 31.1$ & v25 & RR Lyr & 0.43 & $\ldots$ & 0.44 \\
4 & $16: 41: 42.46$ & $36: 28: 08.1$ & & X-ray & & & \\
5 & $16: 41: 41.50$ & $36: 27: 47.8$ & v35 & RR Lyr & 0.32 & $\ldots$ & 0.25 \\
6 & $16: 41: 36.62$ & $36: 26: 35.2$ & v11 & RG & 91.77 & 11.93 & 0.13 \\
7 & $16: 41: 51.99$ & $36: 26: 29.9$ & c & U-V & & & \\
8 & $16: 41: 38.68$ & $36: 25: 37.2$ & v38 & RG & $\ldots$ & 12.12 & 0.07 \\
9 & $16: 41: 48.00$ & $36: 29: 10.1$ & v06 & BL Her & 2.11 & 14.08 & 0.60 \\
10 & $16: 41: 50.95$ & $36: 28: 54.6$ & v17 & RG & 43.04 & 11.98 & 0.38 \\
11 & $16: 41: 46.36$ & $36: 27: 39.9$ & v05 & RR Lyr & 0.38 & 14.77 & 0.42 \\
12 & $16: 41: 46.49$ & $36: 27: 28.1$ & v01 & BL Her & 1.46 & 14.09 & 1.04 \\
13 & $16: 41: 49.07$ & $36: 27: 08.0$ & v34 & RR Lyr & 0.39 & 14.83 & 0.32 \\
14 & $16: 41: 37.11$ & $36: 26: 28.7$ & v07 & RR Lyr & 0.31 & 14.93 & 0.30 \\
15 & $16: 41: 35.51$ & $36: 27: 27.6$ & v42 & RG & $\ldots$ & 11.94 & 0.10 \\
16 & $16: 41: 46.29$ & $36: 27: 37.9$ & v09 & RR Lyr & 0.32 & 14.82 & 0.43 \\
\hline
\end{tabular}

Notes. Variable stars detected with their coordinates (Columns 2 and 3). When available, we list the name in Column 4, and properties of the stars derived by Kopacki et al. (2003) in Columns 5-8: the type of star, the period, the average Vega magnitude in the $V$ band, and the amplitude of the variability $\Delta V$ observed. Star 7 is labeled "c" as first reported by Meinunger (1978) and reused by Kopacki et al. (2003). RG stands for red giant and U-V for UV-bright object as studied by Zinn et al. (1972). X-ray indicates an association with an X-ray source. RR Lyr are short-period ( 0.2 to 2 days) population II pulsating variables often found in GCs. BL Her are a subclass of Type II Cepheid variables with periods between 1 and 8 days.

Table 3

Detected X-ray Sources and Expected Background Sources in M13

\begin{tabular}{lccc}
\hline \hline Radius & 0.62 & 1.69 & $4^{\prime} .2$ \\
\hline Detected & 8 & 15 & 35 \\
Expected & $0.3 \pm 0.3$ & $2.5 \pm 1.6$ & $15.4 \pm 3.9$ \\
Difference & $7.3 \pm 0.3$ & $12.5 \pm 1.6$ & $19.6 \pm 3.9$ \\
\hline
\end{tabular}

possibly indicating a slight hardening of the source in the low luminosity state. In the second observation the median energy is $1.26 \pm 0.16 \mathrm{keV}$.

\subsection{Maximum U-band Magnitude}

We averaged the subtracted images taken between MJD 53419 and 53433 with the FTN, when the star was at maximum luminosity. We also averaged the images between MJD 53472 and 53477 during the minimum. In those two images, the source appears as positive and negative, respectively, compared to the emission of the source in the reference image. In order to obtain the total flux of star 4 between the minimum and maximum states, we subtracted the negative image from the positive image. In Figure 10, we show the reference image and the obtained combination of subtracted images around star 4. An excess is clearly seen, and the error circle of the variable star and the X-ray source overlap, indicating that the two sources are counterparts. One can note that the background is irregular, with many features that show positive and negative peaks. Those are residuals from the subtraction of stars. They can be ruled out as variable stars as the integrated flux in a sufficiently large circle generally remains close to zero and well below the standard deviation of the surrounding background. The shape of the emission is also not as point-like as for star 4 and does not have the expected PSF size.

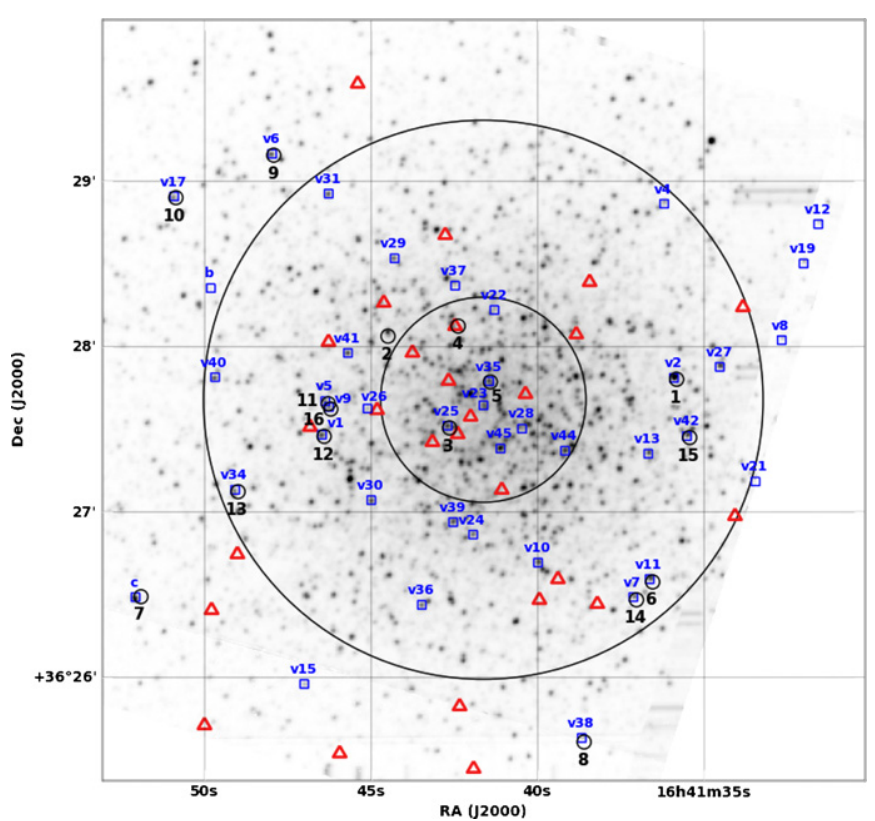

Figure 5. M13 observed with the FTN (reference image). The core and halfmass radii are shown in black. Known variable objects from Kopacki et al. (2003) are plotted as blue squares, X-ray sources detected with Chandra as red triangles, and variable stars detected in this work as black circles.

(A color version of this figure is available in the online journal.)

We studied the stack of subtracted images in the $U$ band with IRAF imexamine in order to determine the magnitude of the star. We first set the zero point with the reference image. We used six isolated and bright reference stars with a known spectral type from SIMBAD. These are not classified as variable and possess $B$ and $V$ magnitude measurements. Based on this information, we extrapolated the expected $U$ magnitude of those stars and scaled the measured $U$ magnitude accordingly. The resulting standard deviation for the set of reference stars is $\sigma_{U}=0.3$. As extinction is low in this field, $E(B-V)=0.02$, it is unlikely to have an effect on this measurement.

Based on the scatter of the background in one subtracted image, the size of the FWHM, and the zero point, we estimate that the $5 \sigma$ detection threshold corresponds to $U=19 \pm 0.3$. We derived a $U$ magnitude of $17.3 \pm 0.3$ for star 4 . The star has not been reported before, so it is likely that the star was fainter and below the detection threshold during its luminosity minimum. The minimum flux would then be more than 1.7 mag lower than the maximum flux. Given the large error bars, we can assume that the measured magnitude in the combination of subtracted images can be directly taken as the maximum magnitude reached by the star. The absolute magnitude is $M_{U}=3.0 \pm 0.3$ if the object is associated with M13.

\subsection{Search for a Counterpart with Hubble}

M13 has been observed with HST for six programs after 1995. We checked the previews of the images acquired and found three programs (proposal IDs 10775, 10349, and 8174) where the region of the X-ray source X6 is covered. The data set with proposal ID 8174 is composed of short exposures only (e.g., $26 \mathrm{~s}$ ) so we focused on the two other data sets obtained with the Advanced Camera for Surveys (ACS) and its Wide Field Camera (WFC) detector. The list of available exposures is given in Table 5. 
Table 4

List of Chandra X-ray Sources in the Field of M13

\begin{tabular}{|c|c|c|c|c|c|c|c|c|}
\hline Source ID & $\begin{array}{c}\text { R.A. } \\
\text { (h:m:s) }\end{array}$ & $\begin{array}{l}\text { Decl. } \\
\text { (d:m:s) }\end{array}$ & $\begin{array}{c}95 \% \text { Error } \\
\left({ }^{\prime \prime}\right)\end{array}$ & $\begin{array}{c}\text { Distance } \\
\left(^{\prime}\right) \\
\end{array}$ & $\begin{array}{c}\text { Net Count Rate } \\
\left(\times 10^{-3} \text { count }^{-1}\right)\end{array}$ & $\begin{array}{c}\text { B1 Counts } \\
(0.3-1.5 \mathrm{keV}) \\
\end{array}$ & $\begin{array}{l}\text { B2 Counts } \\
(1.5-8 \mathrm{keV})\end{array}$ & HR \\
\hline $\mathrm{X} 1$ & $16: 41: 42.015$ & $36: 27: 34.65$ & 0.70 & 0.13 & $0.24 \pm 0.07$ & 10 & 5 & $-0.33 \pm 0.36$ \\
\hline $\mathrm{X} 2$ & $16: 41: 42.676$ & $36: 27: 47.65$ & 0.68 & 0.24 & $0.23 \pm 0.07$ & 11 & 4 & $-0.47 \pm 0.35$ \\
\hline $\mathrm{X} 3$ & $16: 41: 40.366$ & $36: 27: 42.84$ & 0.68 & 0.26 & $0.12 \pm 0.05$ & 4 & 3 & $-0.14 \pm 0.53$ \\
\hline $\mathrm{X} 4$ & $16: 41: 42.401$ & $36: 27: 28.29$ & 0.50 & 0.26 & $0.10 \pm 0.04$ & 6 & 0 & $-1.00 \pm 0.41$ \\
\hline $\mathrm{X} 5$ & $16: 41: 43.160$ & $36: 27: 25.44$ & 0.42 & 0.40 & $0.98 \pm 0.14$ & 22 & 37 & $0.25 \pm 0.18$ \\
\hline X6 & $16: 41: 42.480$ & $36: 28: 07.52$ & 0.15 & 0.48 & $3.19 \pm 0.24$ & 99 & 77 & $-0.12 \pm 0.11$ \\
\hline $\mathrm{X} 7$ & $16: 41: 43.761$ & $36: 27: 57.84$ & 0.08 & 0.51 & $11.05 \pm 0.45$ & 575 & 33 & $-0.89 \pm 0.05$ \\
\hline X8 & $16: 41: 41.078$ & $36: 27: 08.11$ & 0.69 & 0.56 & $0.14 \pm 0.05$ & 6 & 6 & $0.00 \pm 0.41$ \\
\hline X9 & $16: 41: 44.822$ & $36: 27: 37.04$ & 0.26 & 0.64 & $1.66 \pm 0.18$ & 46 & 47 & $0.01 \pm 0.15$ \\
\hline X10 & $16: 41: 38.839$ & $36: 28: 04.53$ & 0.40 & 0.69 & $0.05 \pm 0.03$ & 1 & 2 & $0.33 \pm 0.80$ \\
\hline X11 & $16: 41: 44.622$ & $36: 28: 15.97$ & 0.39 & 0.84 & $0.23 \pm 0.07$ & 12 & 1 & $-0.85 \pm 0.34$ \\
\hline $\mathrm{X} 12$ & $16: 41: 38.439$ & $36: 28: 23.48$ & 0.55 & 0.96 & $0.36 \pm 0.08$ & 14 & 8 & $-0.27 \pm 0.30$ \\
\hline X13 & $16: 41: 46.283$ & $36: 28: 01.74$ & 0.69 & 1.00 & $0.06 \pm 0.04$ & 2 & 4 & $0.33 \pm 0.57$ \\
\hline X14 & $16: 41: 42.775$ & $36: 28: 40.55$ & 1.36 & 1.02 & $0.13 \pm 0.05$ & 2 & 5 & $0.43 \pm 0.52$ \\
\hline $\mathrm{X} 15$ & $16: 41: 46.838$ & $36: 27: 30.93$ & 0.31 & 1.06 & $0.77 \pm 0.12$ & 14 & 30 & $0.36 \pm 0.21$ \\
\hline $\mathrm{X} 16$ & $16: 41: 39.393$ & $36: 26: 35.67$ & 0.58 & 1.17 & $0.27 \pm 0.07$ & 9 & 7 & $-0.12 \pm 0.35$ \\
\hline $\mathrm{X} 17$ & $16: 41: 39.950$ & $36: 26: 28.15$ & 0.31 & 1.26 & $1.19 \pm 0.15$ & 51 & 17 & $-0.50 \pm 0.17$ \\
\hline $\mathrm{X} 18$ & $16: 41: 38.209$ & $36: 26: 26.71$ & 0.12 & 1.41 & $6.39 \pm 0.34$ & 228 & 124 & $-0.30 \pm 0.07$ \\
\hline X19 & $16: 41: 33.832$ & $36: 28: 14.33$ & 0.41 & 1.67 & $0.57 \pm 0.10$ & 19 & 13 & $-0.19 \pm 0.25$ \\
\hline $\mathrm{X} 20$ & $16: 41: 34.074$ & $36: 26: 58.57$ & 0.84 & 1.67 & $0.13 \pm 0.05$ & 6 & 2 & $-0.50 \pm 0.48$ \\
\hline $\mathrm{X} 21$ & $16: 41: 49.019$ & $36: 26: 44.73$ & 0.65 & 1.75 & $0.27 \pm 0.07$ & 7 & 10 & $0.18 \pm 0.34$ \\
\hline $\mathrm{X} 22$ & $16: 41: 42.346$ & $36: 25: 49.57$ & 0.09 & 1.86 & $10.76 \pm 0.45$ & 460 & 124 & $-0.58 \pm 0.06$ \\
\hline $\mathrm{X} 23$ & $16: 41: 45.407$ & $36: 29: 35.47$ & 0.52 & 2.06 & $0.05 \pm 0.03$ & 2 & 1 & $-0.33 \pm 0.80$ \\
\hline $\mathrm{X} 24$ & $16: 41: 49.794$ & $36: 26: 24.47$ & 0.62 & 2.08 & $0.20 \pm 0.06$ & 7 & 5 & $-0.17 \pm 0.41$ \\
\hline $\mathrm{X} 25$ & $16: 41: 41.926$ & $36: 25: 26.92$ & 0.62 & 2.23 & $0.50 \pm 0.10$ & 12 & 19 & $0.23 \pm 0.25$ \\
\hline $\mathrm{X} 26$ & $16: 41: 45.947$ & $36: 25: 32.48$ & 0.68 & 2.31 & $0.27 \pm 0.07$ & 14 & 5 & $-0.47 \pm 0.31$ \\
\hline $\mathrm{X} 27$ & $16: 41: 50.006$ & $36: 25: 42.67$ & 0.65 & 2.59 & $0.14 \pm 0.05$ & 6 & 2 & $-0.50 \pm 0.48$ \\
\hline $\mathrm{X} 28$ & $16: 41: 37.792$ & $36: 25: 05.22$ & 0.20 & 2.70 & $2.84 \pm 0.23$ & 90 & 69 & $-0.13 \pm 0.11$ \\
\hline $\mathrm{X} 29$ & $16: 41: 27.839$ & $36: 27: 34.26$ & 0.90 & 2.78 & $0.06 \pm 0.04$ & 1 & 3 & $0.50 \pm 0.68$ \\
\hline X30 & $16: 41: 41.646$ & $36: 24: 41.70$ & 1.44 & 2.98 & $0.08 \pm 0.04$ & 4 & 1 & $-0.60 \pm 0.60$ \\
\hline X31 & $16: 41: 50.285$ & $36: 25: 13.85$ & 0.25 & 3.00 & $1.30 \pm 0.16$ & 62 & 8 & $-0.77 \pm 0.15$ \\
\hline X32 & $16: 41: 54.727$ & $36: 26: 04.15$ & 0.75 & 3.09 & $0.43 \pm 0.09$ & 8 & 18 & $0.38 \pm 0.27$ \\
\hline X33 & $16: 41: 45.948$ & $36: 24: 22.01$ & 0.62 & 3.42 & $0.69 \pm 0.12$ & 33 & 14 & $-0.40 \pm 0.20$ \\
\hline X34 & $16: 41: 48.727$ & $36: 30: 48.17$ & 0.75 & 3.43 & $0.41 \pm 0.09$ & 13 & 13 & $0.00 \pm 0.28$ \\
\hline X35 & $16: 41: 32.606$ & $36: 30: 37.58$ & 0.37 & 3.46 & $2.17 \pm 0.20$ & 33 & 93 & $0.48 \pm 0.12$ \\
\hline X36 & $16: 41: 36.849$ & $36: 31: 15.09$ & 1.08 & 3.70 & $0.28 \pm 0.08$ & 9 & 10 & $0.05 \pm 0.32$ \\
\hline X37 & $16: 41: 39.239$ & $36: 23: 43.84$ & 0.62 & 3.98 & $1.14 \pm 0.15$ & 51 & 21 & $-0.42 \pm 0.16$ \\
\hline X38 & $16: 41: 30.540$ & $36: 31: 02.52$ & 1.59 & 4.03 & $0.16 \pm 0.06$ & 0 & 10 & $1.00 \pm 0.32$ \\
\hline X39 & $16: 41: 39.846$ & $36: 23: 31.32$ & 1.20 & 4.17 & $0.21 \pm 0.07$ & 12 & 7 & $-0.26 \pm 0.32$ \\
\hline $\mathrm{X} 40$ & $16: 41: 21.629$ & $36: 29: 01.59$ & 1.35 & 4.24 & $0.10 \pm 0.05$ & 1 & 7 & $0.75 \pm 0.46$ \\
\hline $\mathrm{X} 41$ & $16: 42: 02.732$ & $36: 27: 21.40$ & 1.09 & 4.25 & $0.14 \pm 0.05$ & 5 & 8 & $0.23 \pm 0.39$ \\
\hline $\mathrm{X} 42$ & $16: 41: 52.258$ & $36: 23: 23.67$ & 1.13 & 4.79 & $0.11 \pm 0.05$ & 3 & 5 & $0.25 \pm 0.50$ \\
\hline $\mathrm{X} 43$ & $16: 41: 57.158$ & $36: 31: 24.07$ & 1.57 & 4.86 & $0.26 \pm 0.08$ & 6 & 11 & $0.29 \pm 0.34$ \\
\hline $\mathrm{X} 44$ & $16: 41: 32.745$ & $36: 23: 08.08$ & 1.86 & 4.88 & $0.16 \pm 0.06$ & 8 & 21 & $0.45 \pm 0.26$ \\
\hline $\mathrm{X} 45$ & 16:42:03.911 & $36: 25: 27.95$ & 1.60 & 5.00 & $0.10 \pm 0.04$ & 7 & 1 & $-0.75 \pm 0.46$ \\
\hline $\mathrm{X} 46$ & $16: 42: 02.659$ & $36: 30: 59.96$ & 1.17 & 5.37 & $0.35 \pm 0.08$ & 11 & 13 & $0.08 \pm 0.29$ \\
\hline $\mathrm{X} 47$ & $16: 42: 03.380$ & $36: 24: 15.48$ & 0.42 & 5.55 & $4.93 \pm 0.31$ & 126 & 174 & $0.16 \pm 0.08$ \\
\hline
\end{tabular}

Notes. For each source we give its ID, coordinates, and position error (Columns 1-4). We report the distance to the center in Column 5. We then give the net count rate, the number of counts in the two bands $\mathrm{B} 1=0.3-1.5 \mathrm{keV}$ and $\mathrm{B} 2=1.5-8 \mathrm{keV}$, and the hardness ratio $\mathrm{HR}=(\mathrm{B} 2-\mathrm{B} 1) /(\mathrm{B} 1+\mathrm{B} 2)$.

We downloaded the pipeline-reduced images (drz files) and corrected the astrometry with GAIA using three reference stars in the field of view and out of the crowded region of the cluster from the UCAC2 catalog, giving an rms of 0'.0003. The error thus mainly comes from the UCAC2 reference stars, which is $0^{\prime \prime} 04($ at $1 \sigma)$ in this field of view. Images of the region around star 4 are given in Figure 12.

We detected the stars and performed the photometry measurement with IRAF/DAOPHOT. We used six isolated, nonsaturated stars to create the PSF model for each image and applied the task allstar to fit the stars per group of at most 60 stars. The photometry was calibrated using the zero points pro- vided on the ACS Web site ${ }^{12}$ to get Vega magnitudes in each filter. We detected 11 counterparts inside the Chandra error circle of X6 in the F814W image and only 5 in the F658N image. We obtained magnitudes in the four broad bands for seven stars, labeled S1 to S7 according to their distance to X6, which are indicated in Figure 12. They are also reported in the three different CMDs shown in Figure 11. Each diagram was generated with two images taken during the same program.

We note that some spikes from bright stars are apparent in the images (see Figure 12). Unfortunately, these spikes likely

\footnotetext{
12 http://www.stsci.edu/hst/acs/analysis/zeropoints
} 


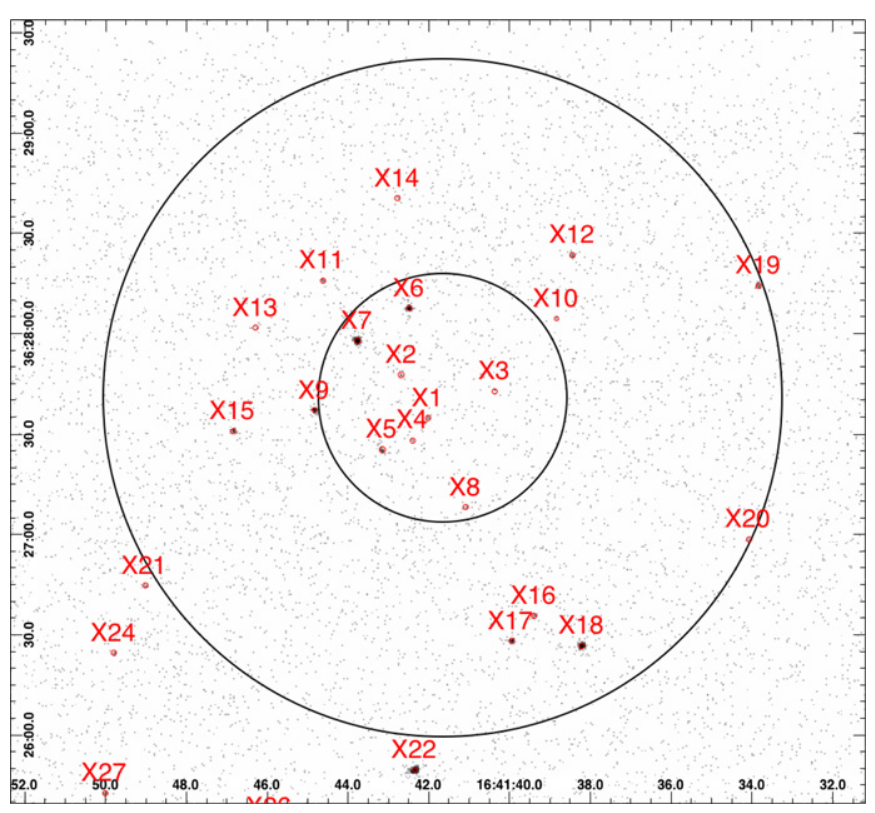

Figure 6. Chandra image of M13 with circles corresponding to the core (0'.62) and half-mass radii (1'.69). Sources are labeled according to their distance to the center of the cluster. Labeled circles correspond to the $3 \sigma$ errors including the absolute pointing accuracy.

(A color version of this figure is available in the online journal.)

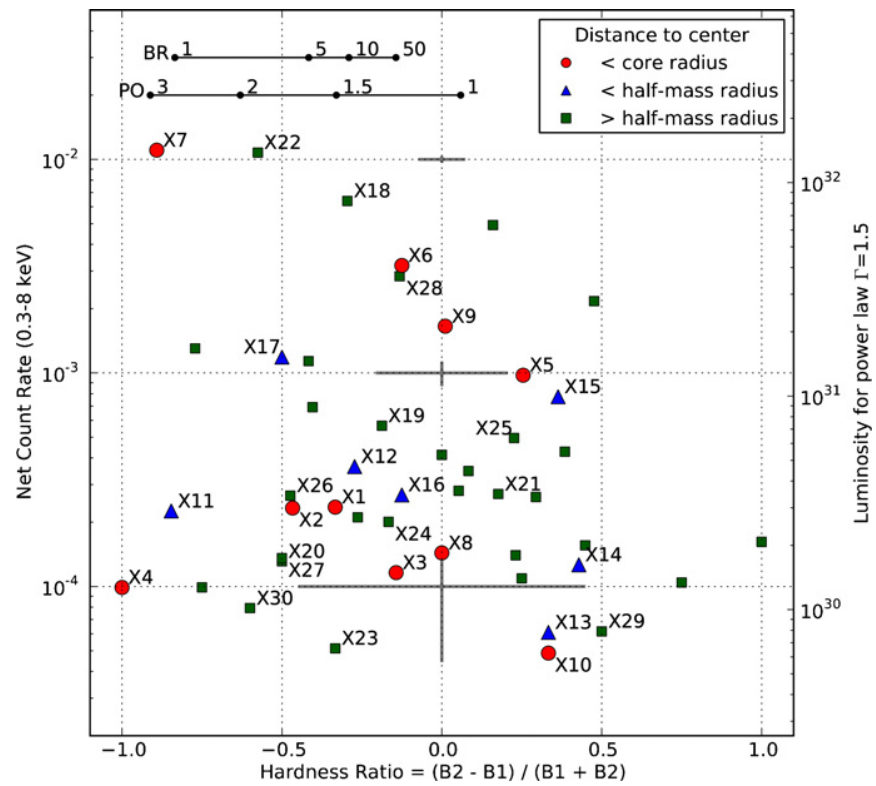

Figure 7. Count rate vs. hardness ratio for the Chandra sources detected in the M13 field of view. For clarity, only the $30 \mathrm{X}$-ray sources closest to the center are labeled. Hardness ratios are computed from the counts in the bands $\mathrm{B} 1=0.3-1.5 \mathrm{keV}$ and $\mathrm{B} 2=1.5-8 \mathrm{keV}$. Black solid lines indicate the hardness ratios corresponding to a power-law model (PO) with different photon indices and a bremsstrahlung emission (BR) with different temperatures $\mathrm{kT}$ in $\mathrm{keV}$. The luminosity for a source in the cluster with a spectrum modeled by a power law of photon index 1.5 is indicated on the right axis. The mean errors for three luminosity levels are indicated by gray bars.

(A color version of this figure is available in the online journal.)

contaminate $\mathrm{S} 1, \mathrm{~S} 2$, and possibly $\mathrm{S} 4$ in the first data set (F625W and $\mathrm{F} 658 \mathrm{~N}$ ), and $\mathrm{S} 4$, and possibly $\mathrm{S} 1$ in the second (F606W and $\mathrm{F} 814 \mathrm{~W}$ ). This may explain the extreme red color of S1 and $\mathrm{S} 2$ in the CMD (F625W, F435W-F625W) which are then bluer and fainter relative to the main sequence in the CMD (F606W, F606W-F814W). Maybe the blue color of S4 in the

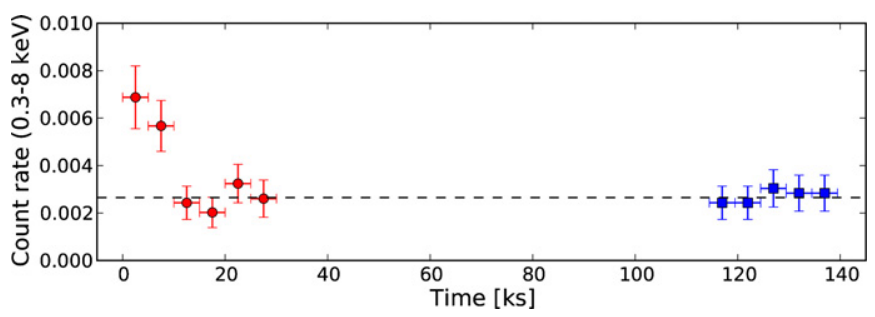

Figure 8. X-ray light curve of Chandra source X6 in M13. The light curve start time is 2006 Mar 9 23:01:13 UT (MJD 53803). The mean level at low luminosity is indicated by a dashed line.

(A color version of this figure is available in the online journal.)

Table 5

Hubble Space Telescope Archived Observations

\begin{tabular}{lcccc}
\hline \hline Prop. ID & Date & MJD & Filter & Exp. time \\
\hline 10349 & 2005 Aug 15 & 53597 & F435W (B) & $2 \times 680 \mathrm{~s}, 1 \times 120 \mathrm{~s}$ \\
& & & F625W (R) & $1 \times 360 \mathrm{~s}, 1 \times 20 \mathrm{~s}$ \\
& & & F658N (H $\alpha)$ & $1 \times 800 \mathrm{~s}, 1 \times 690 \mathrm{~s}$ \\
10775 & \multirow{2}{*}{ 2006 Apr 2 } & \multirow{2}{*}{53827} & F606W (R) & $1 \times 567 \mathrm{~s}$ \\
& & & F814W (I) & $1 \times 567 \mathrm{~s}$
\end{tabular}

CMD (F625W, F435W-F625W) is also due to contamination as the star is faint. There remains, however, a possibility that $\mathrm{S} 1$, S2, and S4 showed color variability.

The CMD (F625W, F658N-F625W) can be used as an indicator of $\mathrm{H} \alpha$ excess. We note that compared to the distribution, $\mathrm{S} 1$ seems to present a $\sim 3 \sigma \mathrm{H} \alpha$ excess. There does not seem to be a strong contamination in the F658N band, and it is likely that it would be compensated or dominated by the contamination in the F625W band.

\section{DISCUSSION}

\subsection{Source 4: A DN Outburst}

Using observations from different observatories in X-ray and optical, we find compelling evidence that variable star 4 (X-ray source X6) is a DN. First, the star shows variability on a timescale of months, consistent with DN outbursts that could last at least 24 days and reached a maximum absolute $U$ magnitude of $M_{U}=3.0 \pm 0.3$ if it is located in M13. We do not have color information, but we note that known DNe in outburst can have $U-V$ values between 0 and -1 (Harrison et al. 2004). Therefore, star 4 is likely to have a maximum magnitude of $M_{V} \gtrsim 3.0$. Previously studied DNe have a maximum absolute magnitude in outburst of $M_{V} \sim 2.7-5.2$ (Harrison et al. 2004). The optical brightenings of variable star 4 would thus be consistent with bright outbursts from a DN located in the core of M13.

The position of this star is consistent with an X-ray source detected using ROSAT, XMM-Newton, and Chandra. The $\mathrm{X}$-ray source has varied in flux between the ROSAT and XMMNewton observations as expected for CVs, but many other $\mathrm{X}$-ray sources show variability. The emission of the Chandra $\mathrm{X}$-ray source is compatible with the expected emission of a DN. Indeed, DNe have luminosities in the range $10^{29}-10^{33} \mathrm{erg} \mathrm{s}^{-1}$ and bremsstrahlung temperatures of $\mathrm{k} T \sim 10 \mathrm{keV}$ (e.g., Verbunt et al. 1997; Baskill et al. 2005). It is thus similar in luminosity to the brightest DN such as U Gem or SU UMa (e.g., Baskill et al. 2005). We note that the temperature we estimated is also consistent with the higher temperatures found for intermediate polars, e.g., 20-30 keV (e.g., Ezuka \& Ishida 1999). A variation in the X-ray count rate of a factor three has been observed with Chandra. This kind of fluctuation is common for 


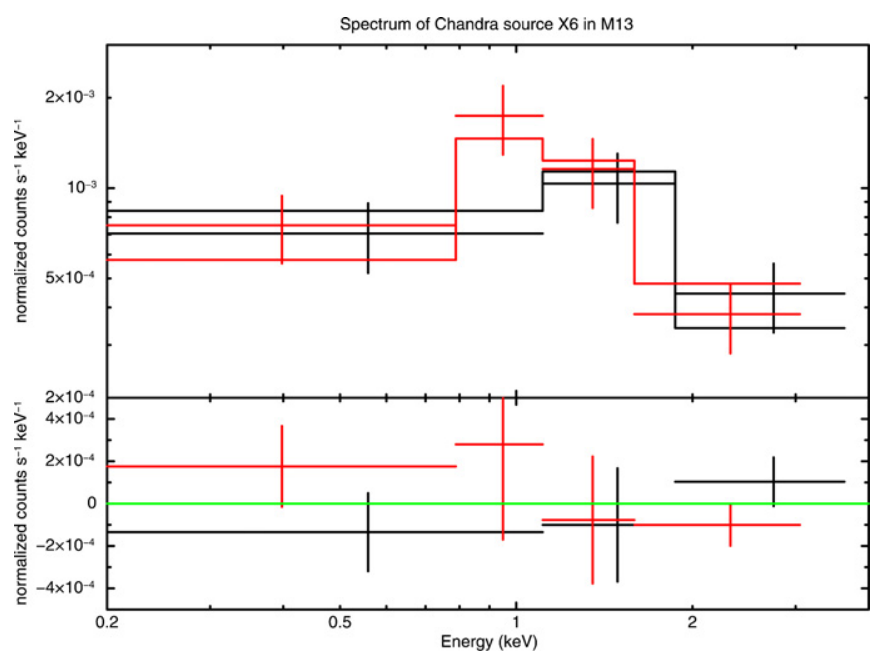

Figure 9. X-ray spectrum of Chandra source X6 in M13. The solid lines are the absorbed bremsstrahlung model. The residuals are indicated in the bottom panel.

(A color version of this figure is available in the online journal.)
CVs or magnetically active binaries. However, the latter generally show an exponential decay of the X-ray flux over a time range of typically $10 \mathrm{ks}$ which is not seen here. We note that this $\mathrm{X}$-ray drop occurred 2 days before $U$-band observations with the FTN which show the star in a bright state. This behavior seems consistent with the X-ray/optical anticorrelation observed for some DNe. For example, SU UMa showed a sudden drop in $\mathrm{X}$-rays by a factor four about half a day after the optical rise (Collins \& Wheatley 2010). The hard X-ray emission of SS Cyg (McGowan et al. 2004) is almost suppressed during the optical outburst. This is somewhat contrary to the possible hardening of the source that we observed after the drop in flux. However, SS Cyg also showed an intermediate state where similar hardening and decrease of the X-ray flux occurred (McGowan et al. 2004). SS Cyg also showed an X-ray flux increase at the beginning of the optical outburst for about half a day, as expected from boundary-layer models (Wheatley et al. 2003). It is possible that the drop in flux we observed corresponds in fact to this increase that precedes the optical outburst.

Finally, using archived data from the $H S T$ we found $\sim 11$ stars inside the Chandra error circle. No bright star was found, thus

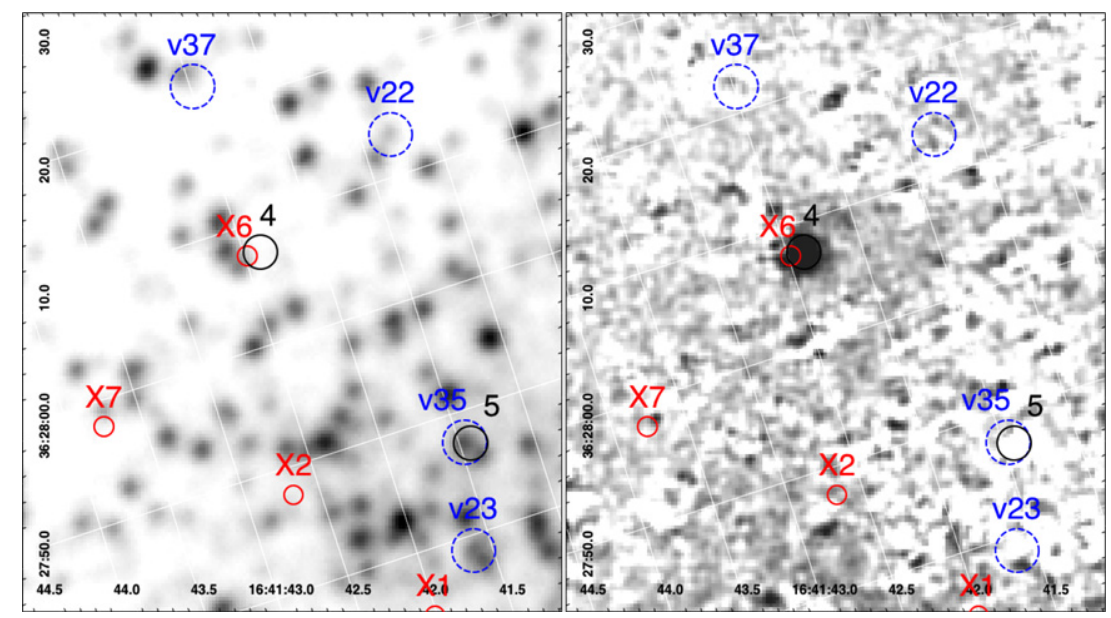

Figure 10. Close-up Faulkes $U$-band image around the Chandra source X6 and the variable star 4. Variable star candidates and X-ray sources are indicated by their 95\% error radius (black and red, respectively), and known variables are indicated with a blue dashed circle of radius 1". 8 for clarity. Left: reference image. Right: combination of subtracted images as presented in the text. We also discuss in the text complex features seen in the background.

(A color version of this figure is available in the online journal.)
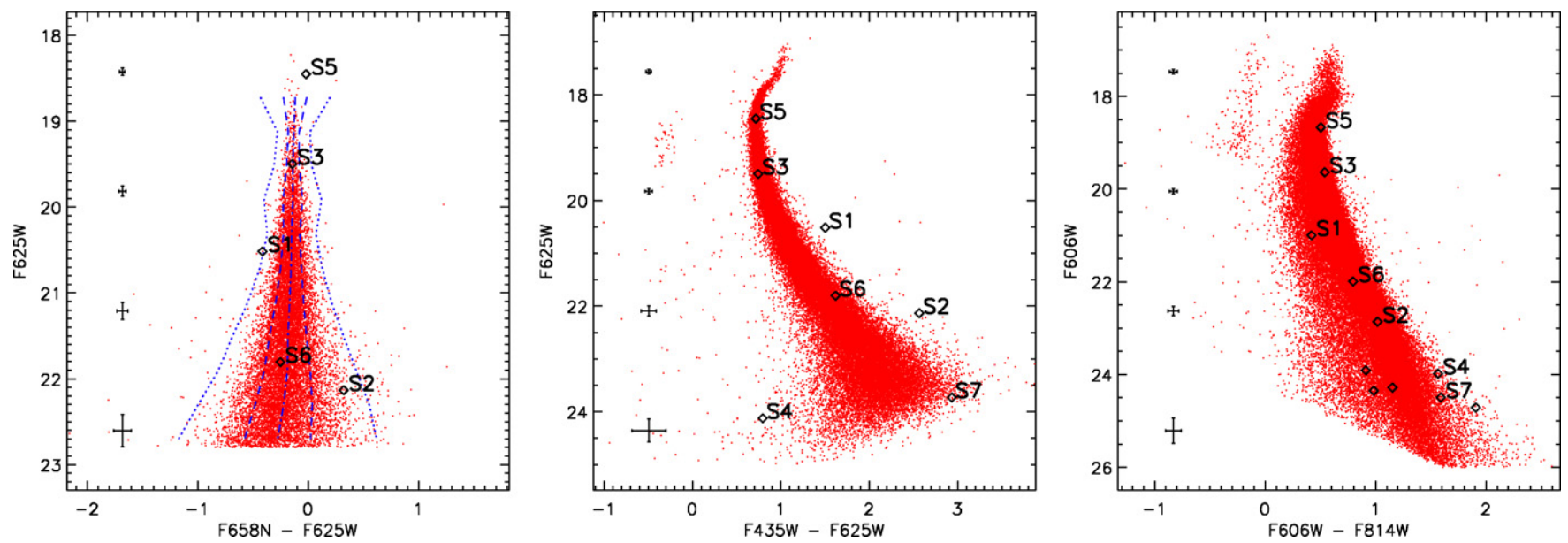

Figure 11. Color-magnitude diagrams of M13 from HST ACS/WFC images. When detected, the stars S1 to S7 shown in Figure 12 are indicated. Error bars are shown on the left of the diagram, which correspond to the mean error for stars in the corresponding magnitude range. In the first diagram, the mean $1 \sigma$ and $3 \sigma$ envelopes of the distribution are shown as dashed lines.

(A color version of this figure is available in the online journal.) 


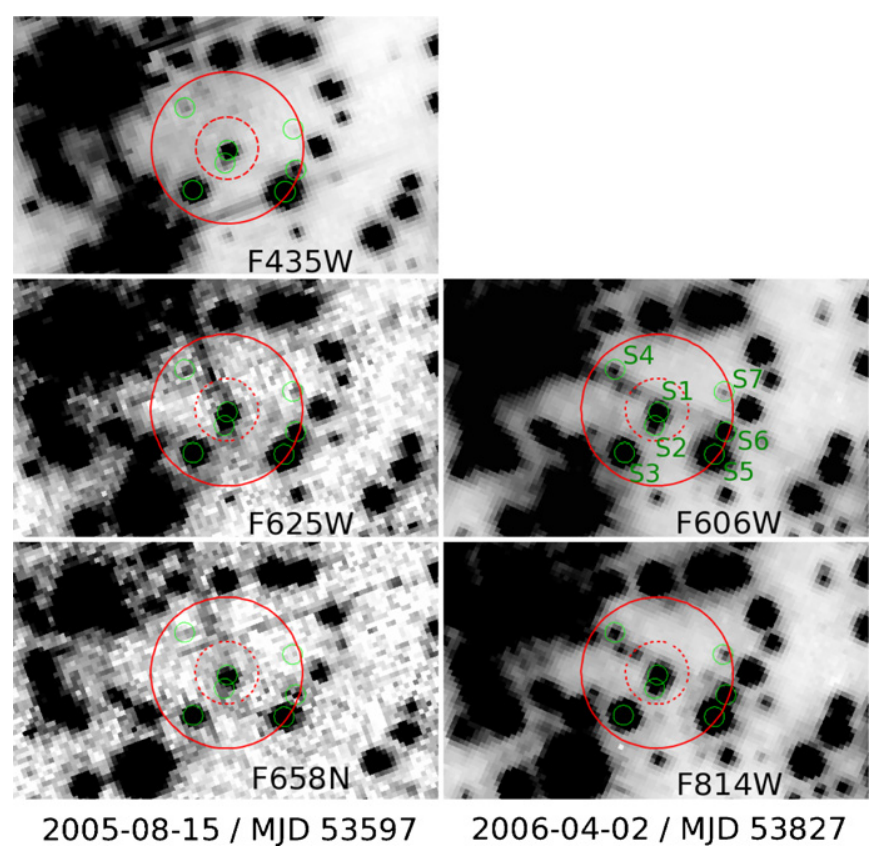

Figure 12. HST ACS/WFC snapshots. Images from the data sets with proposal ID 10349 and 10775 are on the left and right columns, respectively. The size of each image is 4 .' $2 \times 2$ 2.5, north is up, east is left. The $95 \%$ Chandra errors on the position of the candidate $\mathrm{CV}$ are indicated as red circles (small dashed: only detection error; large plain: including absolute pointing error). S1 to S7 are shown with a 2 pixel radius circle.

(A color version of this figure is available in the online journal.)

the object is likely to be in quiescence during those observations. One of the brightest counterparts (S1) presents a possible $\mathrm{H} \alpha$ excess. This kind of emission could come from an accretion disk around a white dwarf and would indicate that $\mathrm{S} 1$ is the true counterpart. However, S1 does not show a blue excess as it is generally expected from $\mathrm{DNe}$, but we note that some $\mathrm{DNe}$ in quiescence in GCs are consistent with main-sequence stars (e.g., CV2 in M22; Pietrukowicz et al. 2005). We note that the star S4 is suspiciously blue in one observation and could have shown color variability; however, it was close to a spike from a bright star and measurements may not be reliable. We could measure magnitudes and colors for the seven brightest counterparts only. Thus, one of the fainter counterparts, for which we could not determine reliable colors, could turn out to be a more convincing association.

If we consider the most studied DNe, their absolute magnitude in quiescence lie in the range $M_{V}=6-10$ (e.g., Patterson 2011). The amplitude of the outburst is generally $d_{M_{V}}=2-6$. DNe with brighter outbursts also tend to have lower amplitudes. At the distance of M13, the quiescent magnitude range would be $V=20.2-24.2$. This outburst being bright, we would then expect a counterpart in quiescence to have a $V$ magnitude in the brighter part of this range. This makes it less likely for fainter stars such as S4 to be the true counterpart, while S1 stands as a good candidate. However, further observations are still needed to locate the true counterpart, with better $\mathrm{S} / \mathrm{N}$ and less contamination from bright stars in order to confirm an $\mathrm{H} \alpha$ excess.

\subsection{Population of $C V s$ in $M 13$}

Based on the encounter rate in M13 and the correlation of this parameter with the number of X-ray sources reported by Pooley \& Hut (2006), we expect about one source within the half-mass radius of M13 above a luminosity threshold of $4 \times 10^{31} \mathrm{erg} \mathrm{s}^{-1}$, where cluster X-ray sources are dominated by CVs. Inside the half-mass radius, we found two X-ray sources above this threshold. One of them is very soft and already reported as a quiescent LMXB candidate (Gendre et al. 2003). The other is the DN discovered in this work. This is consistent with the estimation and strengthens the identification of X6 as a CV.

Ivanova et al. (2006) performed simulations based on different $\mathrm{CV}$ formation channels. Their low-density cluster is the closest to M13, and they estimated that a total of $156 \mathrm{CVs}$ can be formed in such a cluster. From this simulation, about $40 \%$ of the CVs formed are purely primordial CVs (i.e., no dynamical interactions were needed to lead to their formation). Another $46 \%$ of the CVs are also primordial but entered the core of the cluster before the common envelope phase. Only $5 \%$ of the $\mathrm{CVs}$ in the simulation come from formation channels that involve collisions or binary exchange mechanisms. Therefore, the population of CVs in M13 could more closely resemble the population of field CVs than populations in denser GCs, where dynamical effects are believed to modify and enhance the production of CVs.

There have been few detections of DNe in GCs, with only 13 DNe reported so far in the 157 Galactic GCs (e.g., Pietrukowicz et al. 2008; Kaluzny \& Thompson 2009). Based on our knowledge of field CVs, which is extremely biased, it is tempting to try to estimate the expected number of DN outbursts we should have detected in M13, for example following Shara et al. (1996). The DN population constitutes about half of the known CVs listed in the catalog of Downes et al. (2001), which contain mostly field CVs. We could then expect $\sim 78 \mathrm{DNe}$ in M13, based on those rough estimations. The mean duty cycle, or probability of a DN being in outburst at a random epoch, has been found to be $\sim 15 \%$ based on the observation of $21 \mathrm{DNe}$ (Szkody \& Mattei 1984). We can group our observations into five epochs of few days, thus the likelihood of a DN being in quiescence in those five epochs is $0.85^{5}=0.44$. Thus, $\sim 42$ DNe should have been in outburst in at least one of the epochs. It is then harder to estimate the number of outbursts that would have been bright enough to be detected in our observation. Our detection limit at $U \sim 19$ would convert to $V \sim 20\left(M_{V} \sim 5.7\right)$ due to the general blue color of DN outbursts (Harrison et al. 2004). We can then use the distribution of $V$ magnitudes at maximum light from Patterson (2011) based on the observations of $\sim 45 \mathrm{DNe}$, which shows that we could have expected to detect at least two-third of those DNe in outburst at the distance of M13. Finally, $\sim 28 \mathrm{DNe}$ in outburst would have been expected when we detect only one.

However, it is important to note that this estimate is based on several approximations, which makes this number likely to be well overestimated. If we suppose that the mean duty cycle of DNe is $1 \%$ instead of $15 \%$, we then expect $\sim 2 \mathrm{DNe}$ in outburst. We can also suppose that the proportion of DNe is much less than half of all CVs. It would have to be less than $5 \%$ to expect so few $\mathrm{DNe}$ in eruption, as in our observations. Considering that M13 is not an extremely dense GC, with most of its CVs likely being primordial, we may be able to deduce basic information on the composition of the global Galactic CV population. Even if there are large errors in each of our estimates, it seems likely that the mean duty cycle of DNe is lower than $15 \%$, and/or the intrinsic fraction of DNe among CVs may be smaller than catalogs suggest. If the predictions of Ivanova et al. (2006) regarding total numbers of CVs in GCs are correct, then a possible solution to the apparent discrepancy between M13 and the field could be 
that there is a sub-population of CVs that undergo outbursts but have lower luminosities and longer outburst recurrence times than those of the currently known field DNe.

Several population synthesis models already showed that a sub-population of faint CVs with long outburst recurrence times may dominate the total number of CVs (Kolb 1993; Howell et al. 1997; Willems et al. 2005). However, there are still few examples of such a sub-population (e.g., Gänsicke et al. 2009). Deeper surveys of the Galactic Plane are thus needed to catch fainter CVs in order to test this hypothesis. Having observations on timescales of decades could also help to detect outbursts with longer recurrence times. This will maybe be possible through the DASCH project which aims to digitize half a million photographic plates obtained over 100 years in the last century (Grindlay et al. 2009; Laycock et al. 2010; Servillat et al. 2011).

On the other hand, it is possible that our observations were inefficient in detecting DN outbursts. Considering the expected recurrence time of months to years and a duration of at maximum a few weeks for DN outbursts, the optical observation coverage that is likely to detect most of the DN outbursts requires both short scale observations (over few nights) and a recurrence every few weeks, if possible over a year. This kind of survey is thus time consuming, particularly for the large telescopes that are needed if the target is crowded as it is the case for GCs.

Future variability surveys, such as the VISTA Variables in the Via Lactea (VVV; Minniti et al. 2010) public survey and the Large Synoptic Survey Telescope (LSST; Ivezic et al. 2008), will conduct an even denser coverage with $4 \mathrm{~m}$ and $8.4 \mathrm{~m}$ telescopes, respectively. One objective would be to uncover more DNe in GCs and in the field, in order to base our study of CV populations on samples with a decent number of objects.

\section{SUMMARY AND CONCLUSIONS}

We performed a search for optical outbursts in the GC M13. We found one star in M13 which showed variability consistent with outbursts. This star is consistent with being a DN, based on the optical variability and flux, and on the X-ray variability, spectrum, and luminosity. Several possible counterparts were detected in Hubble images, including one with a possible $\mathrm{H} \alpha$ excess and consistent magnitude in quiescence.

The fact that we detect one DN when more have been expected suggests that the known population of local CVs is biased, and short-period, faint, low-duty-cycle (1\%) DNe are likely to dominate the global CV population, as expected from population synthesis models. Their proportion is however not well constrained and future variability surveys will help to better characterize the global CV population.

We thank the anonymous referee whose careful review helped to clarify this paper. M.S. acknowledges supports from NASA/Chandra grants AR9-0013X and GO0-11063X, and NSF grant AST-0909073. This research has made use of the SIMBAD database, operated at CDS, Strasbourg, France.

Facilities: FTN, CXO (ACIS), HST (ACS)

\section{REFERENCES}

Abdo, A. A., et al. 2010, A\&A, 524, A75

Alard, C. 2000, A\&AS, 144, 363

Alard, C., \& Lupton, R. H. 1998, ApJ, 503, 325

Arp, H. C. 1955 , AJ, 60, 317
Baskill, D. S., Wheatley, P. J., \& Osborne, J. P. 2005, MNRAS, 357, 626

Clement, C. M., et al. 2001, AJ, 122, 2587

Collins, D. J., \& Wheatley, P. J. 2010, MNRAS, 402, 1816

Cropper, M. 1990, Space Sci. Rev., 54, 195

Dobrotka, A., Lasota, J.-P., \& Menou, K. 2006, ApJ, 640, 288

Downes, R. A., Webbink, R. F., Shara, M. M., Ritter, H., Kolb, U., \& Duerbeck, H. W. 2001, PASP, 113, 764

Ezuka, H., \& Ishida, M. 1999, ApJS, 120, 277

Gänsicke, B. T., et al. 2009, MNRAS, 397, 2170

Gendre, B., Barret, D., \& Webb, N. A. 2003, A\&A, 403, L11

Grindlay, J., Tang, S., Simcoe, R., Laycock, S., Los, E., Mink, D., Doane, A., \& Champine, G. 2009, in ASP Conf. Ser. 410, Preserving Astronomy's Photographic Legacy: Current State and the Future of North American Astronomical Plates, ed. W. Osborn \& L. Robbins (San Francisco, CA: ASP), 101

Grindlay, J. E. 1999, in ASP Conf. Ser. 157, Annapolis Workshop on Magnetic Cataclysmic Variables, ed. C. Hellier \& K. Mukai (San Francisco, CA: ASP), 377

Harris, W. E. 1996, AJ, 112, 1487

Harrison, T. E., Johnson, J. J., McArthur, B. E., Benedict, G. F., Szkody, P., Howell, S. B., \& Gelino, D. M. 2004, AJ, 127, 460

Hasinger, G., Miyaji, T., \& Schmidt, M. 2005, A\&A, 441, 417

Hong, J., Schlegel, E. M., \& Grindlay, J. E. 2004, ApJ, 614, 508

Hong, J., van den Berg, M., Schlegel, E. M., Grindlay, J. E., Koenig, X., Laycock, S., \& Zhao, P. 2005, ApJ, 635, 907

Howell, S. B., Rappaport, S., \& Politano, M. 1997, MNRAS, 287, 929

Hui, C. Y., Cheng, K. S., \& Taam, R. E. 2010, ApJ, 714, 1149

Hut, P., et al. 1992, PASP, 104, 981

Ivanova, N., Heinke, C. O., Rasio, F. A., Taam, R. E., Belczynski, K., \& Fregeau, J. 2006, MNRAS, 372, 1043

Ivezic, Z., et al. 2008, Serb. Astron. J., 176, 1

Kaluzny, J., \& Thompson, I. B. 2009, Acta Astron., 59, 273

Kolb, U. 1993, A\&A, 271, 149

Kopacki, G., Kołaczkowski, Z., \& Pigulski, A. 2003, A\&A, 398, 541

Laycock, S., Tang, S., Grindlay, J., Los, E., Simcoe, R., \& Mink, D. 2010, AJ, 140,1062

Lewis, F., \& Roche, P. 2009, in Astronomy: Networked Astronomy and the New Media, ed. R. J. Simpson \& D. Ward-Thompson (Bristol: Canopus)

Ludendorff, H. 1905, Publikationen des Astrophysikalischen Observatoriums zu Potsdam, 50, 1

McGowan, K. E., Priedhorsky, W. C., \& Trudolyubov, S. P. 2004, ApJ, 601, 1100

Meinunger, I. 1978, Zentralinstitut fuer Astrophysik Sternwarte Sonneberg Mitteilungen ueber Veraenderliche Sterne, 8, 69

Minniti, D., et al. 2010, New Astron., 15, 433

Osaki, Y. 1996, PASP, 108, 39

Patterson, J. 1994, PASP, 106, 209

Patterson, J. 2011, MNRAS, 411, 2695

Pietrukowicz, P., Kaluzny, J., Thompson, I. B., Jaroszynski, M., SchwarzenbergCzerny, A., Krzeminski, W., \& Pych, W. 2005, Acta Astron., 55, 261

Pietrukowicz, P., Kaluzny, J., Schwarzenberg-Czerny, A., Thompson, I. B., Pych, W., Krzeminski, W., \& Mazur, B. 2008, MNRAS, 388, 1111

Pooley, D., \& Hut, P. 2006, ApJ, 646, L143

Pooley, D., et al. 2003, ApJ, 591, L131

Pretorius, M. L., Knigge, C., \& Kolb, U. 2007, MNRAS, 374, 1495

Roberts, S., Roche, P., \& Ross, R. 2008, in Communicating Astronomy with the Public, ed. L. L. Christensen, M. Zoulias, \& I. Robson (Athens: Eugenides Foundation), 504

Servillat, M., Los, E., Grindlay, J., Tang, S., \& Laycock, S. 2011, in ASP Conf. Ser., ADASS XX, ed. I. N. Evans, A. Accomazzi, D. J. Mink, \& A. H. Rots (San Francisco: ASP), in press (arXiv:1102.4874)

Servillat, M., Webb, N. A., \& Barret, D. 2008a, A\&A, 480, 397

Servillat, M., et al. 2008b, A\&A, 490, 641

Shara, M. M., Bergeron, L. E., Gilliland, R. L., Saha, A., \& Petro, L. 1996, ApJ, 471,804

Skrutskie, M. F., et al. 2006, AJ, 131, 1163

Szkody, P., \& Mattei, J. A. 1984, PASP, 96, 988

Verbunt, F. 2001, A\&A, 368, 137

Verbunt, F., Bunk, W. H., Ritter, H., \& Pfeffermann, E. 1997, A\&A, 327, 602

Watson, M. G., et al. 2009, A\&A, 493, 339

Wheatley, P. J., Mauche, C. W., \& Mattei, J. A. 2003, MNRAS, 345, 49

Willems, B., Kolb, U., Sandquist, E. L., Taam, R. E., \& Dubus, G. 2005, ApJ, 635,1263

Wilms, J., Allen, A., \& McCray, R. 2000, ApJ, 542, 914

Zinn, R. J., Newell, E. B., \& Gibson, J. B. 1972, A\&A, 18, 390 